УДК 621.629

DOI 10.47049/2226-1893-2020-2-142-159

\title{
КОМПЛЕКСНОЕ УПРАВЛЕНИЕ ВЫПУСКНЫМИ ГАЗАМИ СУДОВЫХ ДИЗЕЛЕЙ КАК СПОСОБ ОБЕСПЕЧЕНИЯ ИХ ЭКОЛОГИЧЕСКИХ ПОКАЗАТЕЛЕЙ РАБОТЫ
}

\author{
А.А. Куропятник
} аспирант

Национальный университет «Одесская морская академия»

Аннотация. Приведены результаты исследований по влиянию систем рециркуляиии и перепуска выпускных газов на экологические и экономические показатели работы судового дизеля 16 V32 фирмы Wartsila-Sulzer. Предложено использование комплексной системы управления выпускными газами, сочетающей в себе названные системы. Экспериментально подтверждено, что комплексное управление выпускными газами дизеля 16V32 обеспечивает снижение эмиссии оксидов азота на 31,27-38,23\% при увеличении удельного эффективного расхода топлива на 2,68-1,11\%.

Ключевые слова: экологические показатели дизеля, рециркулячия выпускных газов, перепуск выпускных газов, комплексное управление выпускными газами.

УДК 621.629

DOI 10.47049/2226-1893-2020-2-142-159

\section{КОМПЛЕКСНЕ УПРАВЛІННЯ ВИПУСКНИМИ ГАЗАМИ СУДНОВИХ ДИЗЕЛІВ ЯК СПОСІБ ЗАБЕЗПЕЧЕННЯ ЇХ ЕКОЛОГІЧНИХ ПОКАЗНИКІВ РОБОТИ}

\author{
О.А. Куропятнік \\ аспірант \\ Наџіональний університет «Одеська морська академія»
}

Анотація. Наведено результати досліджень щьодо впливу систем рециркуляиії і перепуску випускних газів на екологічні та економічні показники роботи суднового дизеля 16V32 фірми Wartsila-Sulzer. 3anponoновано використання комплексної системи управління випускними газами, що поєднує в собі названі системи. Експериментально підтверджено, що комплексне управління випускними газами дизеля $16 \mathrm{~V} 32$ забезпечує зниження емісії оксидів азоту на 31,27-38,23 \% при збільшенні питомої ефективної витрати палива на 2,68-1,11\%.

Ключові слова: екологічні показники дизеля, рециркуляція випускних газів, перепуск випускних газів, комплексне управління випускними газами.

(C) Куропятник А.А., 2020 
UDK 621.629

DOI 10.47049/2226-1893-2020-2-142-159

\title{
INTEGRATED MANAGEMENT OF EXHAUST GASES OF MARINE DIESEL ENGINES AS A WAY TO ENSURE THEIR ENVIRONMENTAL PERFORMANCE
}

\author{
O. Kuropyatnyk \\ graduate student
}

National University «Odessa Maritime Academy»

\begin{abstract}
The analysis of ship Diesel engines exhaust gases, assured the reduction of Nitrogen oxide emission into environmental, has been performed. The recirculation exhaust gases system has been analyses, when at $0-21 \%$ of gases return to charge air receiver, as well as the system of exhaust gases by-pass, when 0-10\% of gases return to the exhaust receiver, without preliminary passing through the gas turbine. The result of research on influence of recirculation systems and exhaust gases by-pass to the ecological and economic parameters of ship engine $16 \mathrm{~V} 32$ Wartsila-Sulzer has been provided. The level of exhaust gases recirculation has been changed from 0$21 \%$ and has been controlled on the coefficient excess air ratio during appliance of recirculation and during working of diesel without appliance of recalculated gases. The level of exhaust gases by-pass has been changed into the range $\quad 0-6 \%$ and has been controlled based on the quantity of exhaust gases-general and going to by-pass. Experiments provide that the usage of exhaust gases recirculation into the range of Diesel engine operation service load 16V32 Wartsila-Sulzer 35-95\% assure the decrease of nitrogen oxide emission on 2,4-33,6\%. During this the increasing of specific effective fuel consumption on 3,3-2,2\% is performed. The by-pass system of exhaust gases into the range of Diesel engine charge 16V32 of Wartsila-Sulzer 35-95\% assure the oxide nitrogen emission decreasing on 6,5-17,9\% at simultaneous increasing of specific fuel consumption on 2,7-1,2\%. The appliance of complex system of exhaust gases operation (combining recirculation system and exhaust gases by-pass) assure maximum decrease of oxide nitrogen emission at minimum increase of specific effective fuel consumption. In the meantime for the effective combination of recirculation system and by-pass (that is for the current Diesel engine 16V32 Wartsila-Sulzer comes to $15 \%$ and $6 \%$ ) the oxide nitrogen emission decrease assure on 31,27-38,23\% with respective increasing of specific effective fuel consumption on 2,68-1,11\%. The usage of complex system exhaust gases operation of Diesel engine is the most effective measure to assure its ecological working parameters.
\end{abstract}

Keywords: diesel ecological parameters, exhaust gases recirculation, exhaust gases by-pass, complex operation of exhaust gases. 
Постановка проблемы. Морской транспорт является составляющей частью экономики всех развитых стран, имеющих выход к акватории мирового океана. Согласно «Морскому отчету Организации Объединенных Наций за 2019 год» («United Nations 2018 Maritime Report»), объем морских перевозок в 2018 году достиг 112 млрд. тонн, при этом после мирового кризиса 2008-2010 гг. подтвержден стабильный рост мировой морской торговли. Активное развитие судоходства способствует росту судостроения и двигателестроения. При этом главным генератором энергии, обеспечивающим движение и функционирование судов речного и морского транспорта (вне зависимости их назначения, дедвейта и района плавания), а также жизнедеятельность экипажа является двигатель внутреннего сгорания (ДВС).

Судовые ДВС, вырабатывая полезную энергию (которая передается на гребной винт, обеспечивая движение судна, либо используется для электрического привода вспомогательных механизмов и устройств), являются источниками различного загрязнения окружающей их морской и воздушной среды. К основным продуктам, пагубно влияющим на экологию в результате работы любых тепловых двигателей, относятся нефтяные остатки (загрязненное топливо и масло, дисперсное состояние которых не позволяет топливной аппаратуре осуществить их впрыск в камеру сгорания дизеля или топку котла) и выпускные газы, непосредственно выбрасываемые в атмосферу [1]. Нефтяные остатки (отходы сепарации и фильтрации топлива и масла, а также неразделяемые эмульсии воды и топлива) собираются в специальных накопительных емкостях и впоследствии сдаются на береговые станции рециклинга или утилизации [2]. Выпускные газы, к сожалению, выбрасываются непосредственно в атмосферу, лишь в единичных случаях подвергаясь дополнительной очистке [3].

Анализ последних исследований и публикаций. Для обеспечения экологических показателей судовых дизелей (к которым, прежде всего, относится уровень эмиссии оксидов азота $\mathrm{NO}_{\mathrm{x}}$ с выпускными газами) применяются различные методы. При этом за счет оптимизации процессов смесеобразования, подачи и горения топлива [4], совершенствования конструкции топливной аппаратуры [5], селективного восстановления [6] обеспечивается снижение пиковых температурных нагрузок (что приводит к уменьшению оксидов азота, образующихся в цилиндре дизеля), либо непосредственное удаление $\mathrm{NO}_{\mathrm{x}}$ из потока выпускных газов.

Постановка задачи. Одним из перспективных методов обеспечения экологических показателей дизелей (за счет снижения эмиссии $\mathrm{NO}_{\mathrm{x}}$ ) является управление выпускными газами. При этом возможно обеспечение процессов их рециркуляции (Exhaust Gas Recirculation, EGR) или перепуска (Exhaust Gas Wastegate - EGW). В первом случае часть газового потока возвращается в продувочный ресивер дизеля, во втором - 
направляется в обход газотурбонагнетателя (ГТН) непосредственно в выпускную трубу. Задачей проведенных исследований было определение оптимальных (с точки зрения обеспечения максимального снижения уровня эмиссии $\mathrm{NO}_{\mathrm{X}}$ в выпускных газах при одновременном минимальном повышение удельного расхода топлива) режимов комплексного управления выпускными газами, предусматривающего совместное использование систем EGR и EGW.

Изложение основного материала исследований. Для определения способов адаптивного управления выпускными газами судовых дизелей, при которых обеспечивается максимальное снижение эмиссии оксидов азота при одновременном минимальном изменении энергетических и экономических показателей дизеля, выполнялся комплекс исследований на судовом среднеоборотном дизеле 16V32 фирмы Wartsila-Sulzer. Данные дизели в количестве 4-х единиц использовались в составе пропульсивного комплекса специализированного морского судна водоизмещением 27500 тонн. Особенностью эксплуатации дизеля 16V32 фирмы Wartsila-Sulzer в составе энергетической установки указанного судна является большой эксплуатационный период работы на частичных режимах (в диапазоне мощностей $\left.N_{\text {ереж }}=(0,25-0,75) N_{\text {еном }}\right)$. Дизель укомплектован системой рециркуляции газов низкого давления, в которой газы после газовой турбины ГТН возвращаются в продувочный ресивер дизеля через охладитель наддувочного воздуха. Схема газо-воздушной системы дизеля 16V32 фирмы Wartsila-Sulzer приведена на рис. 1.

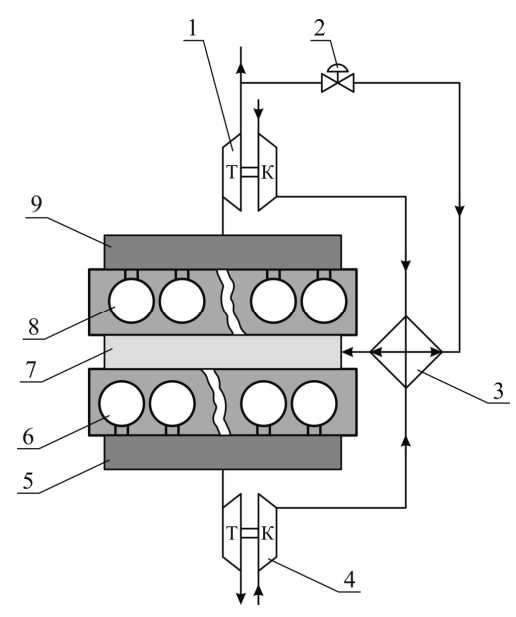

Рис. 1. Схема газо-воздушной системы дизеля $16 V 32$ фирмы Wartsila-Sulzer:

1, 4-ГTН; 2 - клапан системы рециркулящии;

3 - охладитель наддувочного воздуха; 5, 9-выпускной коллектор; 6, 8-рабочие ичилиндры; 7 - ресивер наддувочного воздуха 
V-образная конструкция дизеля предусматривает двустороннее расположение рядов цилиндров 6 и 8, продувочный воздух к которым поступает из общего ресивера 7. Нагнетание воздуха осуществляется компрессорами ГТН 1 и 4, а его охлаждение - в общем охладителе 3. Выпускные газы от цилиндров дизеля 6 и 8 поступают в коллектора 5 и 9 и далее к газовой турбине ГТН 1 и 4 (соответственно). Рециркуляции выпускных газов на дизеле предусмотрена для одного ряда цилиндров 8 и осуществляется с помощью клапана 2 , управляемого микроконтроллером. При этом часть газов (до 30 \%) может возвращаться в продувочный ресивер 7 через охладитель 3. Температура воздуха, поступающего после охладителя 3 в продувочный ресивер и далее в цилиндры дизеля, поддерживалась постоянной. Это обеспечивалось за счет изменения количества забортной воды, поступающего к охладителю 3.

Топливная аппаратура высокого давления позволяла с точностью

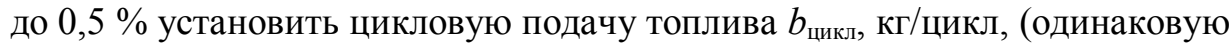
для каждого из цилиндров дизеля), что давало возможность по известным зависимостям $[7 ; 8]$ определить удельный эффективный расход топлива $b_{e}$

$$
b_{e}=\frac{60 n z b_{\text {цикл }}}{N_{e}^{\text {цил }}},
$$

где $n$ - частота вращения коленчатого вала, об/мин;

$z$ - коэффициент тактности;

$N_{e}^{\text {цил }}-$ эффективная цилиндровая мощность, кВт.

Полученное таким образом значение удельного эффективного расхода топлива для каждого из цилиндров усреднялось и дополнительно контролировалось с помощью измерительной аппаратуры, установленной в расходной системе, обеспечивающей подачу топлива к дизелю.

Путем регулирования проходного сечения клапана рециркуляции выпускных газов (позиция 2 на рис. 1) обеспечивалось изменение степени EGR в диапазоне (0-21) \%, которая рассчитывалась по выражению

$$
\delta_{\mathrm{EGR}}=\frac{\alpha_{\mathrm{EGR}}}{\alpha} ;
$$

где $\alpha_{\mathrm{ERG}}, \alpha-$ значение коэффициента избытка воздуха при использовании системы EGR и без ее применения [9].

Значение эмиссии $\mathrm{NO}_{\mathrm{x}}$ в выпускных газах контролировалось с помощью газоанализатора Testo340XL [10].

Результаты измерений и расчетов, позволивших определить величины эмиссии $\mathrm{NO}_{\mathrm{X}}$ в выпускных газах и удельного эффективного расхода топлива $b_{e}$, приведены в таблицах 1 и 2 . 
ВІСНИК

ОДЕСЬКОГО НАЦІОНАЛЬНОГО МОРСЬКОГО УНІВЕРСИТЕТУ № 2 (62), 2020
HERALD

OF THE ODESSA NATIONAL

MARITIME UNIVERSITY № 2 (62), 2020

Таблий 1

Эмиссия $N O_{X}$, гNO $/(\kappa B m \cdot ч)$ судового дизеля 16V32 фирмы Wartsila-Sulzer при различных нагрузках и степени рециркуляичии $\delta_{E G R}$

\begin{tabular}{|c|c|c|c|c|c|c|c|c|}
\hline Нагрузка & \multicolumn{7}{|c|}{ Степень рециркуляции газов $\delta_{\mathrm{EGR}}, \%$} \\
\hline на дизель, \% & 0 & 3 & 6 & 9 & 12 & 15 & 18 & 21 \\
\hline 35 & 8,32 & 7,68 & 7,27 & 6,96 & 6,65 & 6,49 & 6,42 & 6,35 \\
\hline 55 & 8,49 & 8,17 & 7,39 & 7,07 & 6,71 & 6,63 & 6,51 & 6,41 \\
\hline 75 & 8,83 & 8,19 & 7,49 & 7,18 & 6,73 & 6,51 & 6,31 & 6,16 \\
\hline 95 & 8,92 & 8,27 & 7,63 & 7,29 & 7,07 & 6,31 & 6,22 & 5,92 \\
\hline
\end{tabular}

Таблииа 2

Удельный эффективный расход топлива $b_{e}$, г/(кBm·ч) судового дизеля 16V32 фирмы Wartsila-Sulzer

при различных нагрузках и степени рециркуляциии $\delta_{E G R}$

\begin{tabular}{|c|c|c|c|c|c|c|c|c|}
\hline Нагрузка & \multicolumn{7}{|c|}{ Степень рециркуляции газов $\delta_{\mathrm{EGR}}, \%$} \\
\hline на дизель, \% & 0 & 3 & 6 & 9 & 12 & 15 & 18 & 21 \\
\hline 35 & 193,9 & 195,5 & 196,7 & 197,8 & 198,6 & 199,1 & 199,8 & 200,7 \\
\hline 55 & 191,5 & 192,4 & 194,2 & 194,8 & 195,2 & 196,6 & 197,2 & 197,7 \\
\hline 75 & 189,9 & 190,2 & 191,3 & 192,1 & 192,5 & 192,9 & 193,4 & 194,3 \\
\hline 95 & 189,1 & 189,9 & 190,6 & 190,9 & 191,4 & 192,2 & 192,8 & 193,3 \\
\hline
\end{tabular}

По результатам экспериментов, приведенных в таблицах 1 и 2 , построены зависимости, приведенные на рис. 2. При построении комплекса зависимостей $\mathrm{NO}_{\mathrm{X}}=f\left(\delta_{\mathrm{EGR}}\right)$ и $b_{e}=f\left(\delta_{\mathrm{EGR}}\right)$, соответствующих одинаковой нагрузке, первоначально строилась зависимость $\mathrm{NO}_{\mathrm{X}}=f\left(\delta_{\mathrm{EGR}}\right)$, после чего ее нижняя граница принималась за «базовую» линию для построения зависимости $b_{e}=f\left(\delta_{\mathrm{EGR}}\right)$.

Эффективность и экономичность использования системы рециркуляции выпускных газов на различных режимах ее эксплуатации возможно оценить, определив площадь под ломаными $S_{0,35}^{\mathrm{NO} \mathrm{x}_{\mathrm{x}}}=f\left(\delta_{\mathrm{EWG}}\right)$, $S_{0,55}^{\mathrm{NO}_{\mathrm{X}}}=f\left(\delta_{\mathrm{EWG}}\right), \quad S_{0,75}^{\mathrm{NO} \mathrm{X}_{2}}=f\left(\delta_{\mathrm{EWG}}\right), \quad S_{0,95}^{\mathrm{NO} \mathrm{X}_{\mathrm{X}}}=f\left(\delta_{\mathrm{EWG}}\right)$ и $\quad S_{0,35}^{b_{e}}=f\left(\delta_{\mathrm{EWG}}\right), \quad S_{0,55}^{b_{e}}=f\left(\delta_{\mathrm{EWG}}\right)$, $S_{0,75}^{b_{e}}=f\left(\delta_{\mathrm{EWG}}\right), \quad S_{0,95}^{b_{e}}=f\left(\delta_{\mathrm{EWG}}\right)$, где индексы 0,35-0,95 соответствуют нагрузке на дизель. Площадь под зависимостью $S^{\mathrm{NO}_{x}}=f\left(\delta_{\mathrm{EWG}}\right)$ прямо пропорциональна снижению эмиссии оксидов азота с выпускными газами. Чем больше $S^{\mathrm{NO}}$, тем ниже концентрация $\mathrm{NO}_{\mathrm{x}}$ в выпускных газах дизеля. Площадь под зависимостью $S^{b_{e}}=f\left(\delta_{\mathrm{EWG}}\right)$ обратно пропорциональна экономичности работы дизеля. Увеличение $S^{b_{e}}$ приводит к росту удельного эффективного расхода $b_{e}$ топлива на рассматриваемом режиме. 


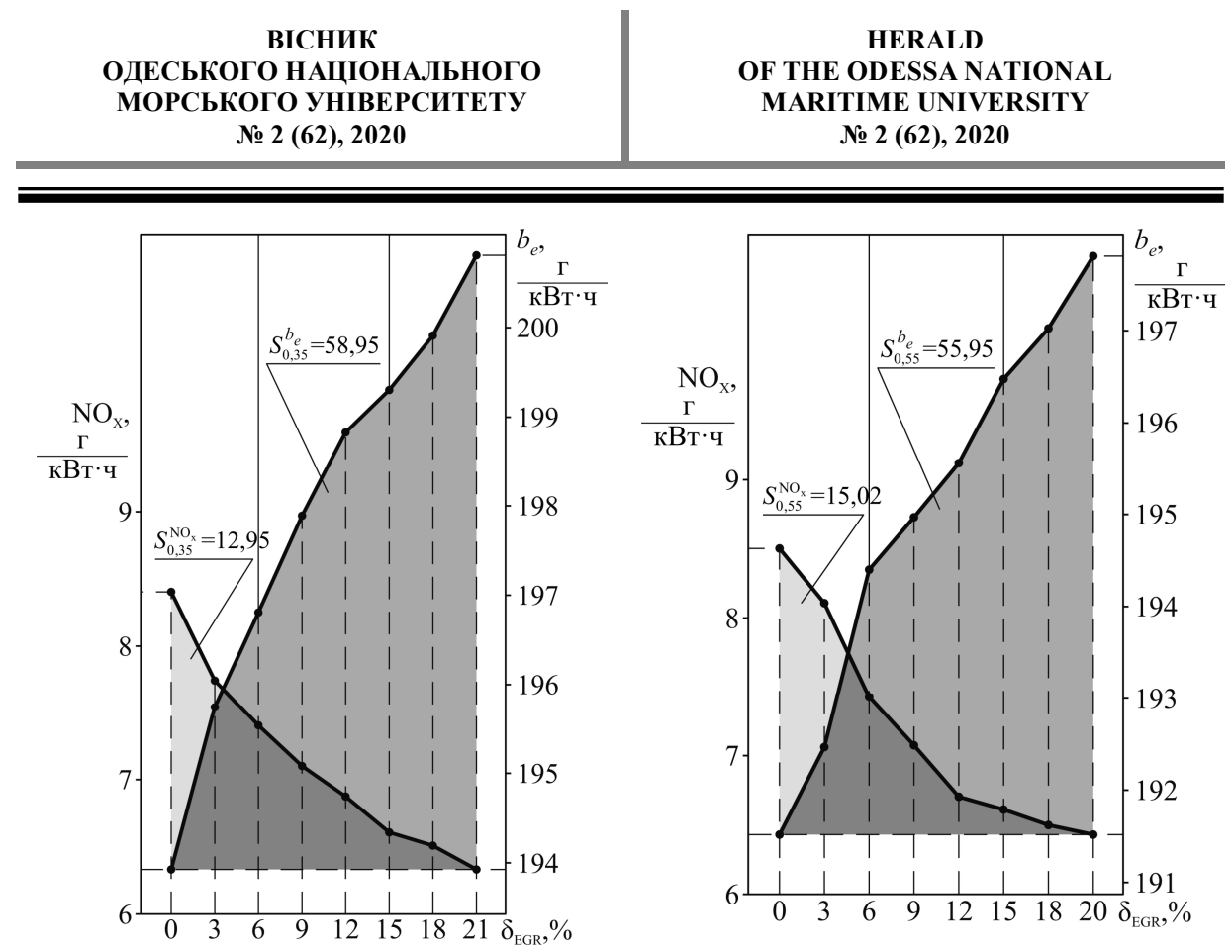

a)

б)

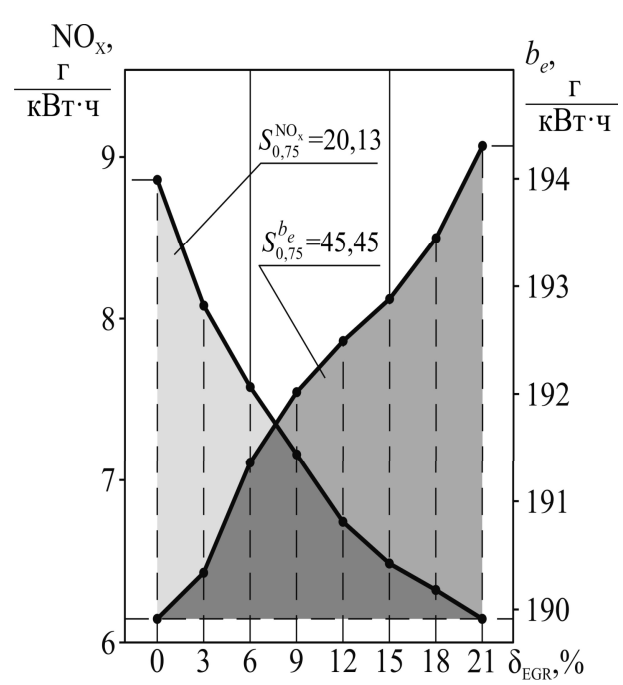

в)

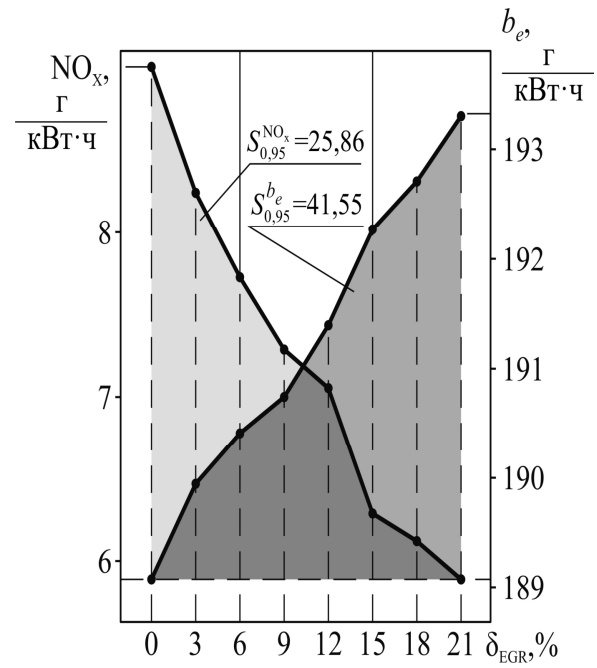

2)

Рис. 2. Влияние степени рецииркуляциии выпускных газов $\delta_{E G R}$ на эмиссию $\mathrm{NO}_{X}$ и удельный эффективный расход топлива $b_{e}$ при различных нагрузках судового дизеля 16V32 фирмы Wartsila-Sulzer:

$a-0,35 N_{\text {еном }} ; \sigma-0,55 N_{\text {еном }} ; в-0,75 N_{\text {еном }} ; 2-0,95 N_{\text {еном }}$ 
ВІСНИК

ОДЕСЬКОГО НАЦІОНАЛЬНОГО

МОРСЬКОГО УНІВЕРСИТЕТУ

№ 2 (62), 2020
HERALD

OF THE ODESSA NATIONAL

MARITIME UNIVERSITY № 2 (62), 2020

Для изучения комплексного управления выпускными газами судовых дизелей с целью снижения эмиссии $\mathrm{NO}_{\mathrm{X}}$ приведенная на рис. 1 газовоздушная система дополнительно комплектовалась оборудованием, обеспечивающим процесс перепуска газов (рис. 3). При этом регулирующий клапан 10 обеспечивал перепуск выпускных газов непосредственно в газовыпускную трубу без их расширения в газовой турбине 1 . Количество выпускных газов (общее $G_{\Sigma}$ и идущее на перепуск $G_{\text {п }}$ ) определялось в точках K2 и K2 (соответственно) с помощью расходомера MT100S, позволяющего выполнять измерения в газовых потоках с температурой до $454{ }^{\circ} \mathrm{C}$.

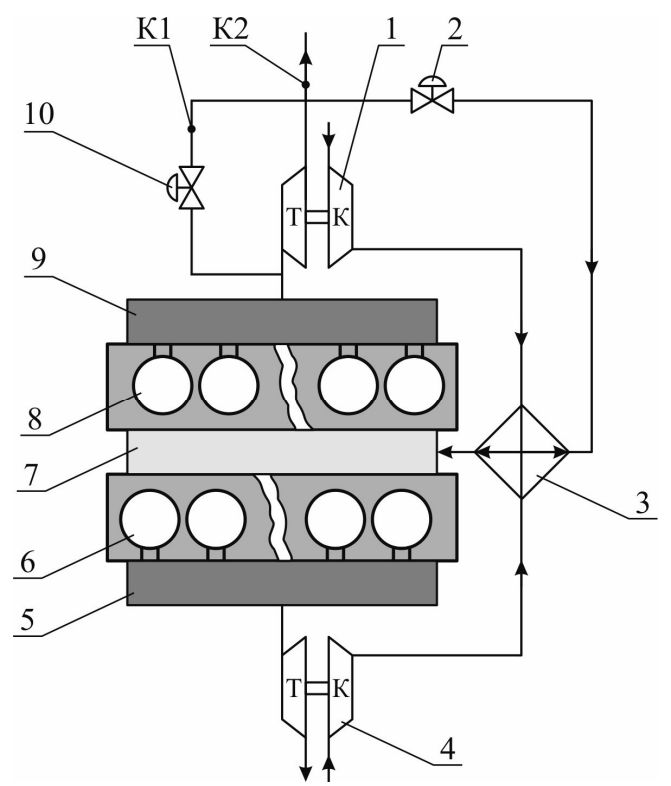

Рис. 3. Схема газо-воздушной системы дизеля 16V32/40

фирмы Wartsila-Sulzer с дополнительным перепуском выпускных газов:

1, 4-ГТН; 2 -клапан системы рециикуляици;

3 - охладитель наддувочного воздуха; 5, 9-выпускной коллектор;

6, 8-рабочие цилиндры; 7 -ресивер наддувочного воздуха;

10 - клапан перепуска газов (клапан $E W G$ );

K1, К2 - точки контроля потока газов

Степень перепуска газов $\delta_{\text {EWG }}$ рассчитывалась по выражению

$$
\delta_{\mathrm{EWG}}=\frac{G_{\text {п }}}{G_{\Sigma}} \cdot 100 \%
$$


Таблий 3

Эмиссия $N O_{X}$, гNO $/(\kappa B m \cdot ч)$ судового дизеля 16V32 фирмы Wartsila-Sulzer при различных нагрузках и степени перепуска газов $\delta_{E W G}$

\begin{tabular}{|c|c|c|c|c|c|c|}
\hline Нагрузка & \multicolumn{7}{|c|}{ Степень перепуска газов $\delta_{\text {ЕWG }} \%$} \\
\cline { 2 - 7 } на дизель, \% & 0 & 2 & 4 & 6 & 8 & 10 \\
\hline 35 & 8,32 & 8,17 & 8,04 & 7,96 & 7,83 & 7,78 \\
\hline 55 & 8,49 & 8,32 & 8,12 & 7,90 & 7,82 & 7,72 \\
\hline 75 & 8,83 & 8,55 & 8,18 & 7,96 & 7,72 & 7,62 \\
\hline 95 & 8,92 & 8,53 & 7,88 & 7,62 & 7,43 & 7,32 \\
\hline
\end{tabular}

Таблий 4

Удельный эффективный расход топлива $b_{e}$, г/(кBm·ч) судового дизеля 16V32 Wartsila-Sulzer

при различных нагрузках и степени перепуска газов $\delta_{E W G}$

\begin{tabular}{|c|c|c|c|c|c|c|}
\hline Нагрузка & \multicolumn{7}{|c|}{ Степень перепуска газов $\delta_{\text {ЕWG, }} \%$} \\
\cline { 2 - 7 } на дизель, \% & 0 & 2 & 4 & 6 & 8 & 10 \\
\hline 35 & 193,9 & 195,8 & 196,8 & 197,4 & 198,6 & 199,4 \\
\hline 55 & 191,5 & 193,2 & 194,2 & 194,8 & 195,2 & 195,4 \\
\hline 75 & 189,9 & 191,7 & 192,2 & 192,8 & 192,9 & 193,1 \\
\hline 95 & 189,1 & 190,3 & 190,8 & 191,1 & 191,3 & 191,5 \\
\hline
\end{tabular}

Анализ результатов, приведенных в таблицах 3,4 и рис. 4 , характеризует диапазон изменения степени перепуска выпускных газов $\delta_{\mathrm{EWG}}=6-8 \%$ как наиболее оптимальный с точки зрения экологических и экономических показателей работы. В этом диапазоне для нагрузок на дизель 35-95 \% происходит снижение эмиссии $\mathrm{NO}_{\mathrm{X}}$ на 4,33-16,7 \% (при максимальном снижении эмиссии $\mathrm{NO}_{\mathrm{X}}$ на 6,5-17,9\% при условии $\delta_{\mathrm{EWG}}=10 \%$ и ивеличение удельного расхода топлива $b_{e}$ для того же интервала нагрузок на 1,04-2,37\% (при минимальном увеличении $b_{e}$ на 0,63-0,97 \% при условии $\delta_{\mathrm{EWG}}=2 \%$ ).

Учитывая изложенное, для определения комплексного влияния систем EGR и EWG на экологические и экономические показатели работы дизеля для дальнейших исследований в качестве оптимального принималось значение $\delta_{\mathrm{EWG}}=6 \%$. Эту величину обеспечивало постоянное положение клапана перепуска (позиция 10 на рис. 3). Изменением положения клапана рециркуляции выпускных газов (позиция 2 на рис. 3) обеспечивались различные значения $\delta_{\mathrm{EGR}}$. Результаты комплексного влияния систем EGR и EWG на экологические и экономические показатели работы дизеля 16V32 фирмы Wartsila-Sulzer приведены в таблицах 5 и 6 и показана на рис. 5. 


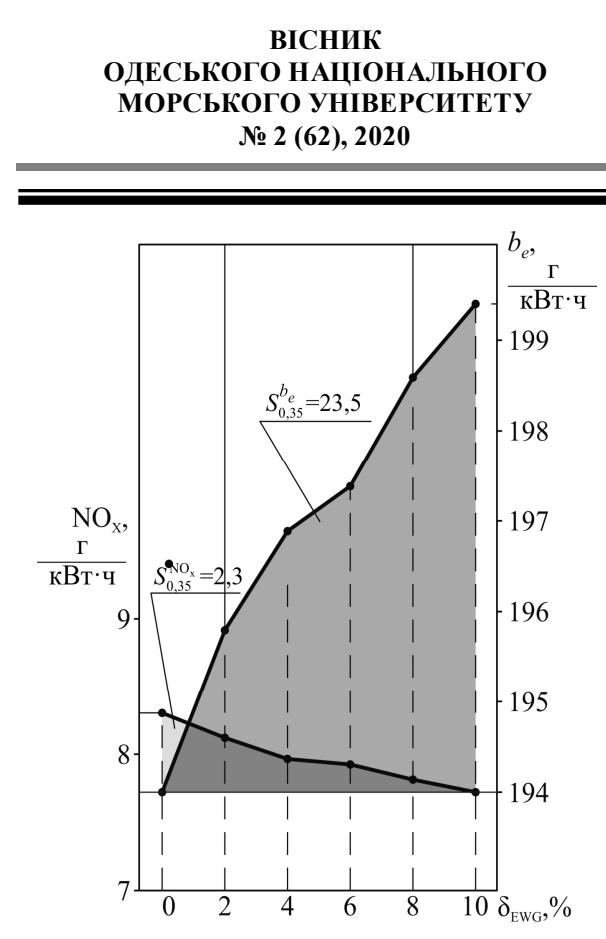

a)

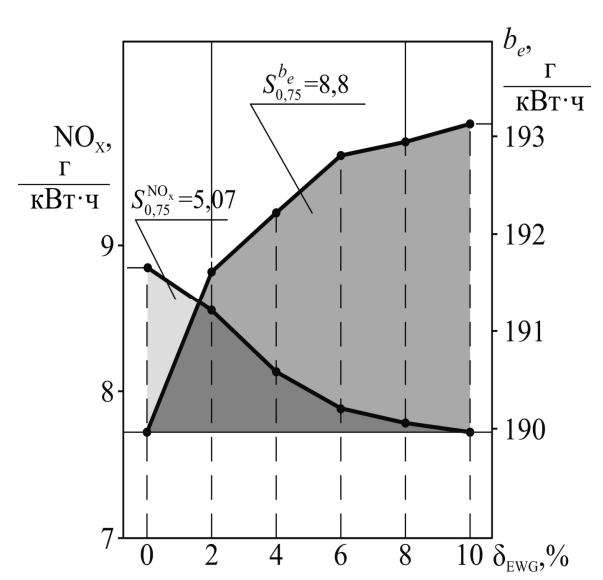

в)
HERALD

OF THE ODESSA NATIONAL

MARITIME UNIVERSITY

№ 2 (62), 2020

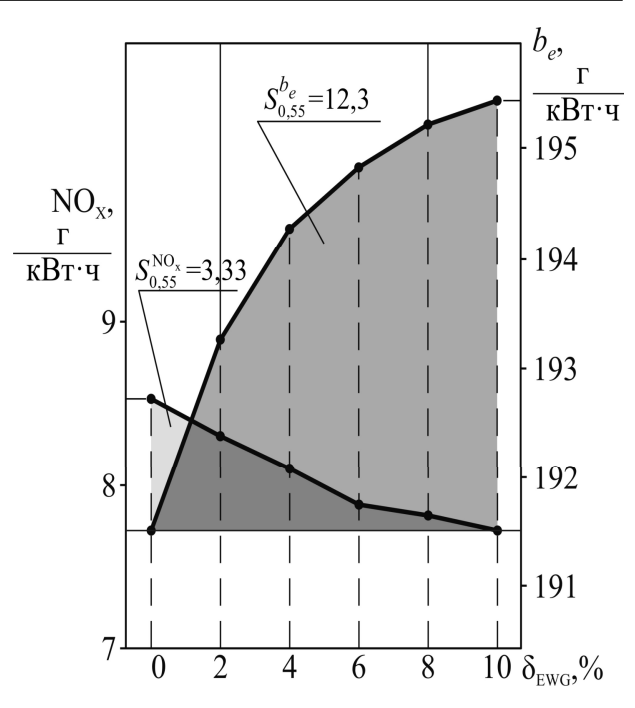

б)

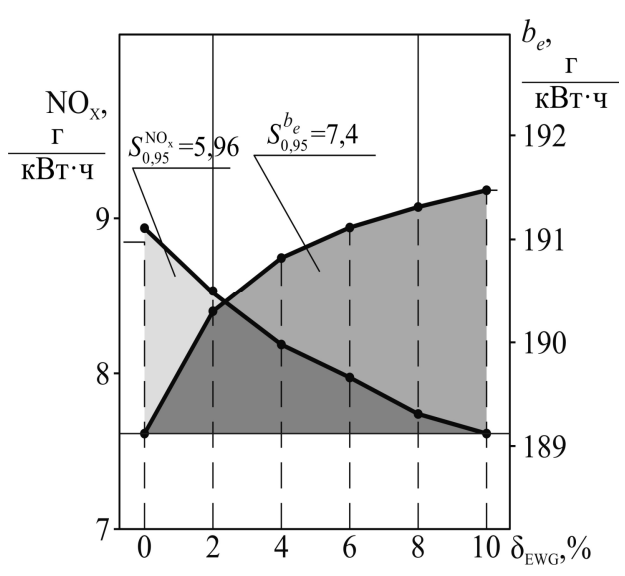

2)

Рис. 4. Влияние $\delta_{E W G}$ на эмиссию $N O_{X}$

и удельный эффективный расход топлива $b_{e}$

при различных нагрузках судового дизеля 16V32 Wartsila-Sulzer:

$$
a-0,35 N_{\text {еном }} ; \sigma-0,55 N_{\text {еном }} ; \text { в }-0,75 N_{\text {еном }} ; 2-0,95 N_{\text {еном }}
$$

Интегральная оценка влияния различных способов управления выпускными газами на экологические и экономические показатели работы дизеля может быть выполнена по значению площади под зависимостями $S^{\mathrm{NO}_{\mathrm{x}}}=f\left(\delta_{\mathrm{EGR}}\right), S^{\mathrm{NO}_{\mathrm{x}}}=f\left(\delta_{\mathrm{EWG}}\right), S^{b_{e}}=f\left(\delta_{\mathrm{EGR}}\right), S^{b_{e}}=f\left(\delta_{\mathrm{EWG}}\right)$. Значение данных величин приведено в таблице 7. 
ВІСНИК

ОДЕСЬКОГО НАЦІОНАЛЬНОГО

МОРСЬКОГО УНІВЕРСИТЕТУ

№ 2 (62), 2020
HERALD

OF THE ODESSA NATIONAL

MARITIME UNIVERSITY

№ 2 (62), 2020

Таблий 5

Эмиссия $N O_{X}, 2 N O_{X} /(\kappa B m \cdot ч)$ судового дизеля $16 \mathrm{~V} 32$ фирмь

Wartsila-Sulzer при комплексном управлении выпускными газами (для оптимального значения степени перепуска газов $\delta_{E W G}$ и различных значениях степени рецииркуляции газов $\delta_{E G R}$ ) при различных нагрузках

\begin{tabular}{|c|c|c|c|c|c|c|c|c|}
\hline \multirow{2}{*}{$\begin{array}{c}\text { Нагрузка } \\
\text { на дизель, \% }\end{array}$} & \multicolumn{8}{|c|}{ Степень рециркуляции газов $\delta_{\mathrm{EGR}}, \%$} \\
\hline & 0 & 3 & 6 & 9 & 12 & 15 & 18 & 21 \\
\hline 35 & 8,32 & 7,43 & 7,07 & 6,61 & 6,12 & 5,71 & 5,52 & 5,42 \\
\hline 55 & 8,49 & 7,73 & 7,17 & 6,87 & 6,45 & 6,12 & 5,76 & 5,53 \\
\hline 75 & 8,83 & 7,62 & 6,85 & 6,53 & 6,27 & 5,58 & 5,18 & 4,83 \\
\hline 95 & 8,92 & 7,41 & 7,13 & 6,26 & 5,81 & 5,51 & 4,75 & 4,43 \\
\hline
\end{tabular}

Таблииа 6

Удельный эффективный расход топлива $b_{e}$, г/(кBm·ч) судового дизеля 16V32 фирмы Wartsila-Sulzer при комплексном управлении выпускными газами (для оптимального значения степени перепуска газов $\delta_{E W G}$ и различных значениях степени рещиикулящии газов $\delta_{E G R}$ ) при различных нагрузках

\begin{tabular}{|c|c|c|c|c|c|c|c|c|}
\hline Нагрузка & \multicolumn{7}{|c|}{ Степень рециркуляции газов $\delta_{\mathrm{EGR},} \%$} \\
\cline { 2 - 9 } на дизель, \% & 0 & 3 & 6 & 9 & 12 & 15 & 18 & 21 \\
\hline 35 & 193,9 & 195,3 & 196,2 & 196,8 & 197,5 & 197,6 & 198,3 & 198,7 \\
\hline 55 & 191,5 & 192,2 & 193,4 & 193,8 & 194,2 & 194,5 & 194,8 & 195,2 \\
\hline 75 & 189,9 & 190,1 & 190,8 & 191,4 & 191,8 & 192,3 & 192,5 & 192,8 \\
\hline 95 & 189,1 & 189,5 & 190,3 & 190,5 & 190,9 & 191,2 & 191,6 & 191,8 \\
\hline
\end{tabular}

Увеличение $S^{\mathrm{NO}_{\mathrm{x}}}$ в случае варианта управления выпускными газами EGR+EWG свидетельствует о большей эффективности данного метода.

В таблицах 8, 9 и на рис. 6 приведено процентное изменение значений эмиссии $\mathrm{NO}_{\mathrm{x}}$ с выпускными газами и удельного эффективного расхода топлива $b_{e}$ для различных способов управления выпускными газами. 
Интегральная оченка влияния способов управления выпускными газами на экологические и экономические показатели работы дизеля 16V32 фирмы Wartsila-Sulzer

\begin{tabular}{|c|c|c|c|c|}
\hline \multirow{2}{*}{$\begin{array}{c}\text { Способ управления } \\
\text { выпускными газами }\end{array}$} & \multicolumn{4}{|c|}{ Нагрузка на дизель, \% } \\
\hline & $0,35 N_{\text {еном }}$ & $0,55 N_{\text {еном }}$ & $0,75 N_{\text {еном }}$ & $0,95 N_{\text {еном }}$ \\
\hline \multicolumn{5}{|c|}{$\begin{array}{l}\text { Снижение эмиссии } \mathrm{NO}_{\mathrm{x}}-\text { как площадь под зависимостью } \\
\qquad S^{\mathrm{NO}_{\mathrm{x}}}=f\left(\delta_{\mathrm{EGR}}\right), S^{\text {No }}=f\left(\delta_{\mathrm{EWG}}\right)\end{array}$} \\
\hline $\begin{array}{l}\text { Рециркуляция выпуск- } \\
\text { ных газов, EGR }\end{array}$ & 12,95 & 15,02 & 20,13 & 25,86 \\
\hline $\begin{array}{l}\text { Перепуск выпускных } \\
\text { газов, EWG }\end{array}$ & 2,3 & 3,33 & 5,07 & 5,94 \\
\hline $\begin{array}{l}\text { Комплексное управле- } \\
\text { ние выпускными газа- } \\
\text { ми, EGR+EWG }\end{array}$ & 22,02 & 29,85 & 32,63 & 37,13 \\
\hline \multicolumn{5}{|c|}{$\begin{array}{c}\text { Увеличение удельного эффективного расхода топлива } b_{e}-\text { как площадь под } \\
\text { зависимостью } S^{b_{e}}=f\left(\delta_{\mathrm{EGR}}\right), S^{b_{e}}=f\left(\delta_{\mathrm{EWG}}\right)\end{array}$} \\
\hline $\begin{array}{l}\text { Рециркуляция выпуск- } \\
\text { ных газов, EGR }\end{array}$ & 58,95 & 55,95 & 45,45 & 41,85 \\
\hline $\begin{array}{l}\text { Перепуск выпускных } \\
\text { газов, EWG }\end{array}$ & 23,5 & 12,3 & 8,8 & 7,4 \\
\hline $\begin{array}{l}\text { Комплексное управле- } \\
\text { ние выпускными газа- } \\
\text { ми, EGR+EWG }\end{array}$ & 38,1 & 29,85 & 27,6 & 24,5 \\
\hline
\end{tabular}

Таблийа 8

Снижение эмиссии $\mathrm{NO}_{X}, \%$, судового дизеля 16V32 фирмы Wartsila-Sulzer при различных способах управления выпускными газами

\begin{tabular}{|l|c|c|c|c|}
\hline \multirow{2}{*}{$\begin{array}{c}\text { Способ управления } \\
\text { выпускными газами }\end{array}$} & \multicolumn{4}{|c|}{ Нагрузка на дизель, \% } \\
\cline { 2 - 5 } & $0,35 N_{\text {еном }}$ & $0,55 N_{\text {еном }}$ & $0,75 N_{\text {еном }}$ & $0,95 N_{\text {еном }}$ \\
\hline $\begin{array}{l}\text { Рециркуляция выпускных } \\
\text { газов, } \delta_{\mathrm{EGR}}=15 \%\end{array}$ & 21,99 & 21,91 & 26,27 & 29,26 \\
\hline $\begin{array}{l}\text { Перепуск выпускных } \\
\text { газов, } \delta_{\mathrm{EWG}}=6 \%\end{array}$ & 4,33 & 5,42 & 9,85 & 14,57 \\
\hline $\begin{array}{l}\text { Комплексное управление } \\
\text { выпускными газами, } \\
\delta_{\mathrm{EGR}}=15 \%+\delta_{\mathrm{EWG}}=6 \%\end{array}$ & 31,37 & 27,92 & 36,81 & 38,23 \\
\hline
\end{tabular}




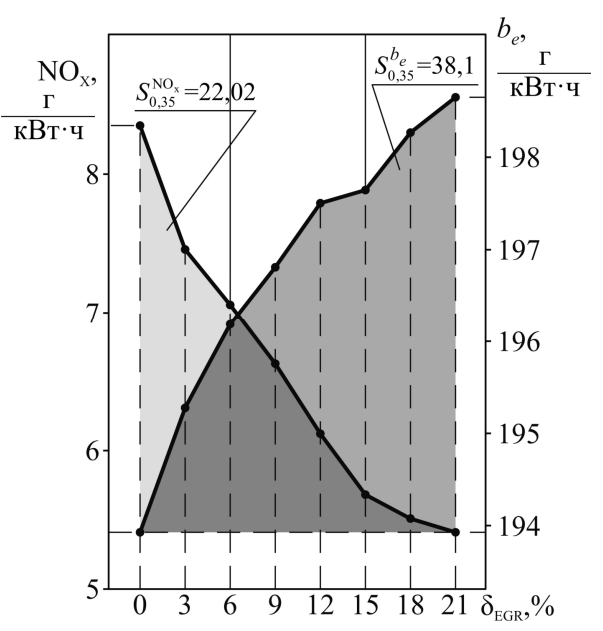

a)

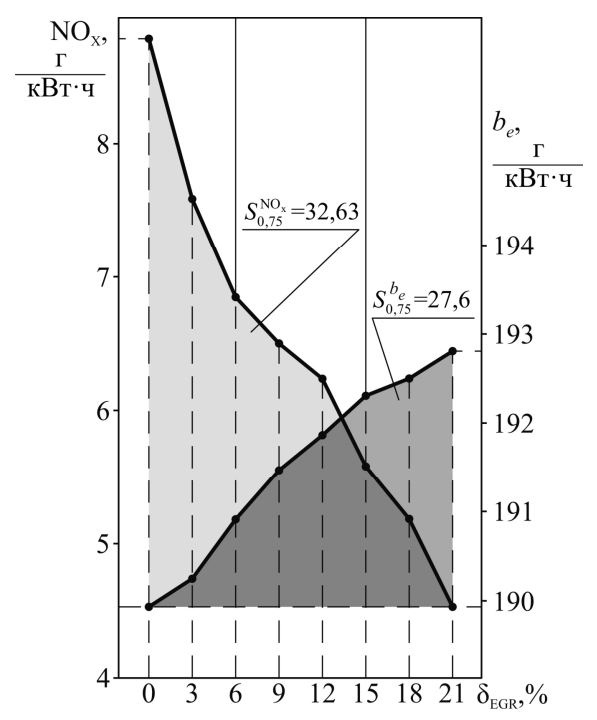

в)

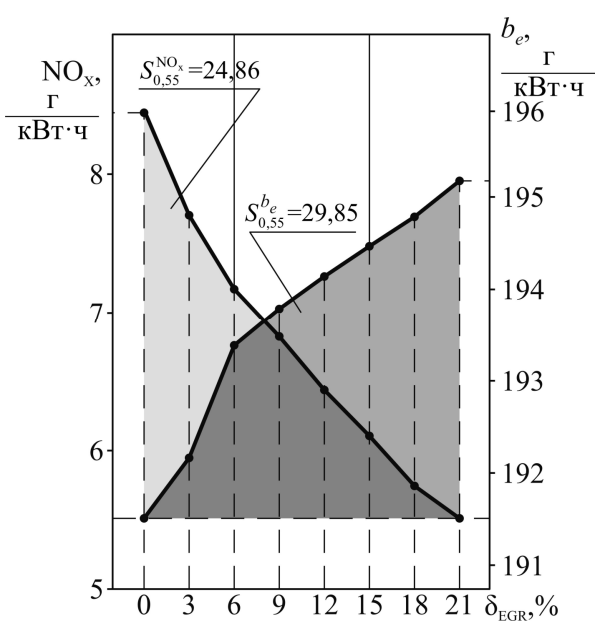

б)

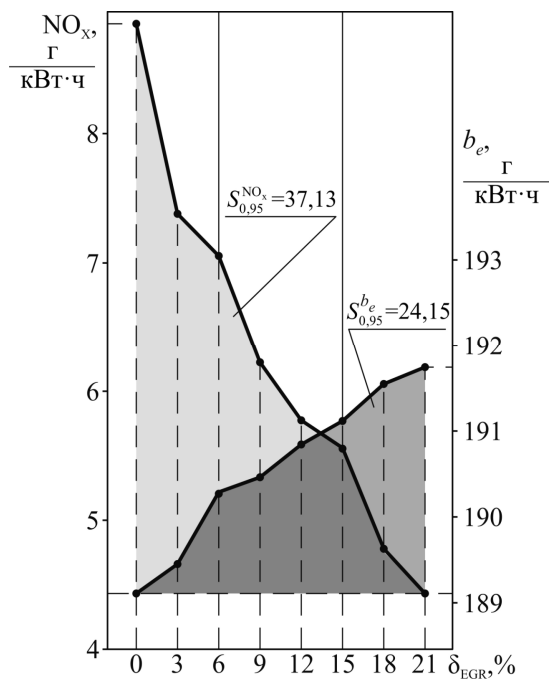

2)

Рис. 5. Эмиссия $N O_{X}$ и удельный эффективныий расход топлива $b_{e}$ судового дизеля 16V32 фирмы Wartsila-Sulzer при комплексном управлении выпускными газами (для оптимального значения степени перепуска газов $\delta_{E W G}$ и различных значениях степени рециркуляции газов $\left.\delta_{E G R}\right)$ при различных нагрузках:

$$
a-0,35 N_{\text {еном }} ; \sigma-0,55 N_{\text {еном }} ; \text { в }-0,75 N_{\text {еном }} ; 2-0,95 N_{\text {еном }}
$$


ВІСНИК

ОДЕСЬКОГО НАЦІОНАЛЬНОГО

МОРСЬКОГО УНІВЕРСИТЕТУ

№ 2 (62), 2020
HERALD

OF THE ODESSA NATIONAL

MARITIME UNIVERSITY

№ 2 (62), 2020

Таблииа 9

Увеличение удельного эффективного расхода топлива $b_{e}, \%$, судового дизеля 16V32 фирмы Wartsila-Sulzer при различных способах управления выпускными газами

\begin{tabular}{|l|c|c|c|c|}
\hline \multicolumn{1}{|c|}{$\begin{array}{c}\text { Способ управления } \\
\text { выпускными газами }\end{array}$} & \multicolumn{4}{|c|}{ Нагрузка на дизель, \% } \\
\cline { 2 - 5 } & $0,35 N_{\text {еном }}$ & $0,55 N_{\text {еном }}$ & $0,75 N_{\text {еном }}$ & $0,95 N_{\text {еном }}$ \\
\hline $\begin{array}{l}\text { Рециркуляция выпуск- } \\
\text { ных газов, } \delta_{\text {EGR }}=15 \%\end{array}$ & 2,68 & 2,66 & 1,58 & 1,64 \\
\hline $\begin{array}{l}\text { Перепуск выпускных } \\
\text { газов, } \delta_{\text {EWG }}=6 \%\end{array}$ & 2,84 & 2,04 & 1,69 & 1,27 \\
\hline $\begin{array}{l}\text { Комплексне управле- } \\
\text { ние выпускными газами, } \\
\delta_{\mathrm{EGR}}=15 \%+\delta_{\mathrm{EWG}}=6 \%\end{array}$ & 1,91 & 1,57 & 1,26 & 1,11 \\
\hline
\end{tabular}

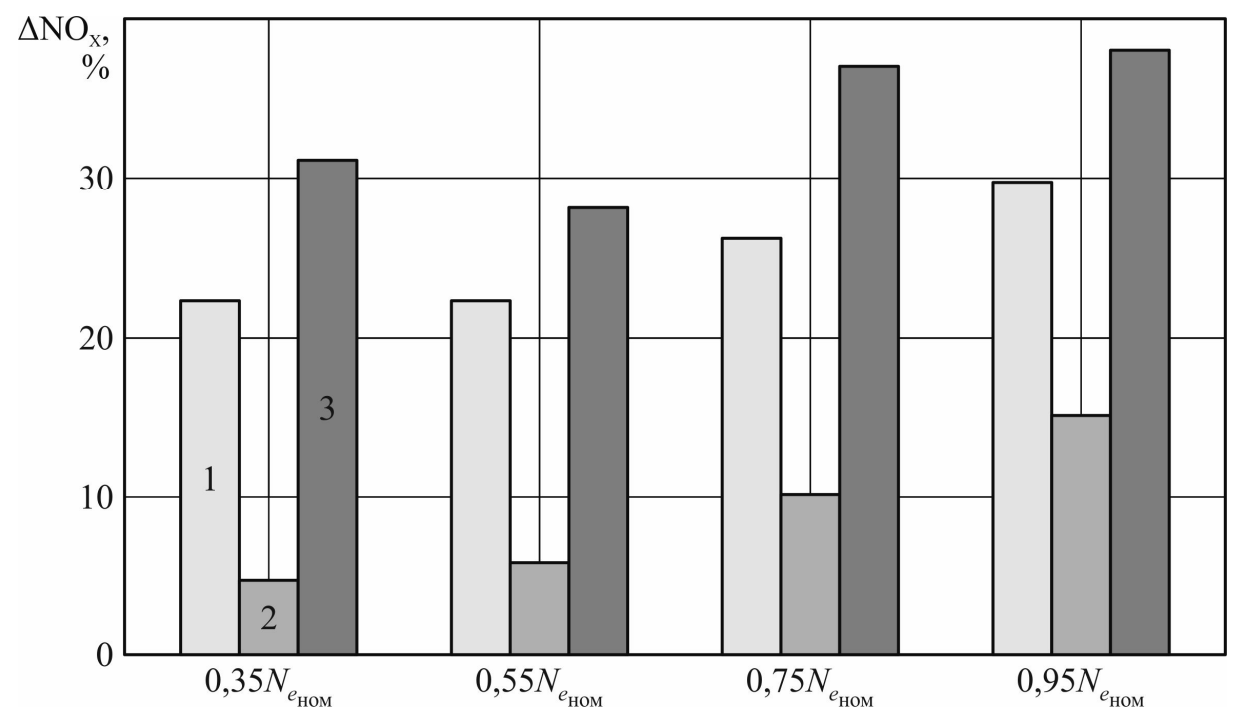

a)

Рис. 6. Снижение эмиссии $\mathrm{NO}_{X}, \%$, (a)

и увеличение удельного эффективного расхода топлива $b_{e}, \%$, (б) судового дизеля 16V32 фирмы Wartsila-Sulzer при различных способах управления выпускными газами:

$$
1-\delta_{E G R}=15 \% ; 2-\delta_{E W G}=6 \% ; 3-\delta_{E G R}=15 \%+\delta_{E W G}=6 \%
$$




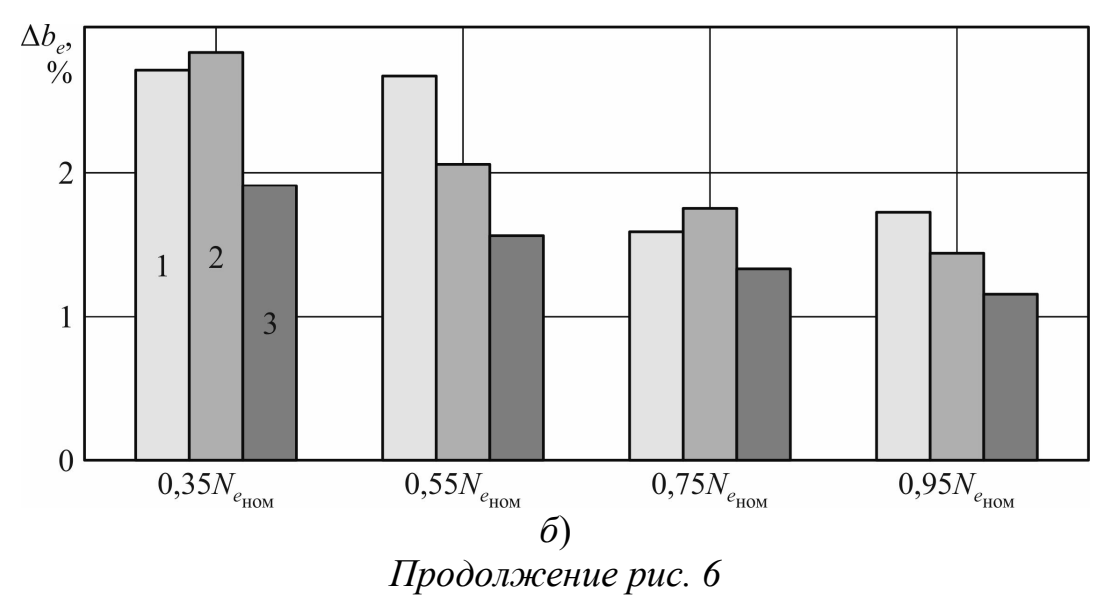

Выводы и перспективы дальнейших исследований. Результаты проведенных исследований позволяют сделать следующие выводы.

Системы управления выпускными газами (к которым относятся системы рециркуляции и перепуска выпускных газов), использующиеся на судах речного и морского флота для обеспечения экологических показателей работы судовых дизелей, способствуют снижению эмиссии оксидов азота (как основного токсичного компонента выпускных газов) в окружающую среду.

Система рециркуляции выпускных газов в диапазоне нагрузок судового дизеля 16V32 фирмы Wartsila-Sulzer 35-95\% обеспечивает снижение эмиссии оксидов азота на 2,4-33,6 \%. При этом происходит соответственное увеличение удельного эффективного расхода топлива на $3,3-2,2 \%$.

Система перепуска выпускных газов в диапазоне нагрузок судового дизеля 16V32 фирмы Wartsila-Sulzer 35-95 \% обеспечивает снижение эмиссии оксидов азота на 6,5-17,9 \%. При этом происходит соответственное увеличение удельного эффективного расхода топлива на 2,7-1,2 \%.

Применение комплексной системы управления выпускными газами (объединяющей системы рециркуляции и перепуска выпускных газов) обеспечивает максимальное снижение эмиссии оксидов азота при минимальном повышении удельного эффективного расхода топлива. При этом для оптимального сочетания степени рециркуляции $\delta_{\mathrm{EGR}}$ и перепуска $\delta_{\mathrm{EWG}}$ (которое для рассмотренного дизеля 16V32 фирмы Wartsila-Sulzer составляет $\delta_{\mathrm{EWG}}=15 \%$ и $\delta_{\mathrm{EWG}}=6 \%$ ) достигается снижение эмиссии оксидов азота на $31,27-38,23 \%$ при соответственном увеличении удельного эффективного расхода топлива на 2,68-1,11\%.

Использование комплексной системы управления выпускными газами судовых дизелей является наиболее эффективным способом обеспечения их экологических показателей работы. 


\section{СПИСОК ЛІТЕРАТУРИ}

1. Sagin, S.V. Cavitation Treatment of High-Viscosity Marine Fuels for Medium-Speed Diesel Engines / S.V. Sagin, V.G. Solodovnikov // Modern Applied Science. 2015. Vol. 9. № 5. P. 269-278. DOI:10.5539/mas.v9n5p269.

2. Sagin, S.V. Marine Slow-Speed Diesel Engine Diagnosis with View to Cylinder Oil Specification / S.V. Sagin, O.V. Semenov / American Journal of Applied Sciences. 2016. Vol. 13. Iss. 5. P. 618-627. DOI: 10.3844/ajassp. 2016.618.627.

3. Куропятнік О.А. Зниження емісії оксидів азоту суднових дизелів методом перепуску випускних газів / О.А. Куропятнік // Вісник Одеськ. наи. мор. ун-ту, 2018. Bun. 4(57). C. 98-108.

4. Sagin, S.V. The Use of Exhaust Gas Recirculation for Ensuring the Environmental Performance of Marine Diesel Engines / S.V. Sagin, O.A. Kuropyatnyk // OUR SEA : International Journal of Maritime Science \& Technology. June 2018. Vol. 65. № 2. P. 78-86. doi.org/10.17818/NM/ 2018/2.3.

5. Zablotsky, Yu.V. Enhancing Fuel Efficiency and Environmental Specifications of a Marine Diesel When using Fuel Additives / Yu.V. Zablotsky, S.V. Sagin // Indian Journal of Science and Technology, Published by Indian Society of Education and Environment. December 2016. Vol. 9. Iss. 46. P. 353-362. DOI: $10.17485 / i j s t / 2016 / v 9 i 46 / 107516$.

6. Kuropyatnyk, O.A. Exhaust Gas Recirculation as a Major Technique Designed to Reduce NOx Emissions from Marine Diesel Engines / O.A. Kuropyatnyk, S.V. Sagin // OUR SEA: International Journal of Maritime Science \& Technology. 2019. Vol. 66. Iss. 1. P. 1-9. doi.org/10.17818/ NM/2019/1.1.

7. Yeryganov, O. Features of the fastest pressure growth point during compression stroke / O. Yeryganov, R. Varbanets // Diagnostyka. 2018. Vol. 19. № 2. P.71-76. http://dx.doi.org/ 10.29354/ diag/ 89729.

8. Sagin, S.V. Estimation of Operational Properties of Lubricant Coolant Liquids by Optical Methods / S.V. Sagin, V.G. Solodovnikov // International Journal of Applied Engineering Research. 2017. Vol. 12. Num. 19. P. 8380-8391.

9. Kuropyatnyk, O.A. Reducing the emission of nitrogen oxides from marine diesel engines / O.A. Kuropyatnyk // Materials of the International Conference "Scientific research of the SCO countries: synergy and integration». January 25, 2020. Part 2. Beijing, PRC. P. 154-160. DOI. 10.34660/INF. 2020.24.53689. 
10. Sagin, S.V. Application of the system of recirculation of exhaust gases for the reduction of the concentration of nitric oxides in the exhaust gases of the ship diesels / S.V. Sagin, A.A. Kuropyatnik // American Scientific Journal, 2017. № 15. Iss. 2. P. 67-71.

\section{REFERENCES}

1. Sagin, S.V., Solodovnikov, V.G. (2015). Cavitation Treatment of High-Viscosity Marine Fuels for Medium-Speed Diesel Engines. Modern Applied Science, vol. 9, no. 5, pp. 269-278. DOI:10.5539/ mas. v9n5p269.

2. Sagin, S.V., Semenov, O.V. (2016). Marine Slow-Speed Diesel Engine Diagnosis with View to Cylinder Oil Specification. American Journal of Applied Sciences, vol. 13, no. 5, pp. 618-627. DOI: 10.3844/ajassp.2016.618.627.

3. Kuropyatnyk, O.A. (2018). Znyzhennya emisiyi oksydiv azotu sudnovyx dyzeliv metodom perepusku vypusknyx gaziv [Reduction of nitrogen oxides of marine diesel by exhaust gas bypass]. Visnyk Odeskogo nacionalnogo morskogo unyversytetu, no. 4(57), pp. 98-108.

4. Sagin, S.V., Kuropyatnyk, O.A. (2018). The Use of Exhaust Gas Recirculation for Ensuring the Environmental Performance of Marine Diesel Engines. OUR SEA: International Journal of Maritime Science \& Technology, vol. 65, no 2, pp. 78-86. doi.org/ 10.17818/NM/2018/2.3.

5. Zablotsky, Yu.V., Sagin, S.V. (2016). Enhancing Fuel Efficiency and Environmental Specifications of a Marine Diesel When using Fuel Additives. Indian Journal of Science and Technology, vol. 9 , no. 46, pp. 353-362. DOI: 10.17485/ijst/2016/v9i46/107516.

6. Kuropyatnyk, O.A., Sagin, S.V. (2019). Exhaust Gas Recirculation as a Major Technique Designed to Reduce NOx Emissions from Marine Diesel Engines. OUR SEA: International Journal of Maritime Science \& Technology, vol. 66, no. 1, pp. 1-9. doi.org/ 10.17818/ NM/2019/1.1.

7. Yeryganov, O., Varbanets, R. (2018). Features of the fastest pressure growth point during compression stroke. Diagnostyka, vol. 19, no. 2, pp. 71-76. http://dx.doi.org/ 10.29354/diag/89729.

8. Sagin, S.V., Solodovnikov, V.G. (2017). Estimation of Operational Properties of Lubricant Coolant Liquids by Optical Methods. International Journal of Applied Engineering Research, vol. 12, no. 19, pp. 8380-8391. 
ВІСНИК

ОДЕСЬКОГО НАЦІОНАЛЬНОГО

МОРСЬКОГО УНІВЕРСИТЕТУ

№ 2 (62), 2020
HERALD

OF THE ODESSA NATIONAL

MARITIME UNIVERSITY

№ 2 (62), 2020

9. Kuropyatnyk, O.A. (2020). Reducing the emission of nitrogen oxides from marine diesel engines. Materials of the International Conference «Scientific research of the SCO countries: synergy and integration», Beijing, PRC, January 25, 2020, vol. 2, pp. 154-160. DOI. 10.34660/INF. 2020.24.53689.

10. Sagin, S.V., Kuropyatnyk, O.A. (2017) Application of the system of recirculation of exhaust gases for the reduction of the concentration of nitric oxides in the exhaust gases of the ship diesels. American Scientific Journal, no. 15, iss. 2, pp. 67-71.

Стаття надійшла до редакиії 24.01.2020

Посилання на статтю: Куропятник А.А. Комплексное управление выпускными газами судовых дизелей как способ обеспечения их экологических показателей работы // Вісник Одеського національного морського університету: Зб. наук. праць, 2020. № 2(62). C. 142-159. DOI 10.47049/2226-18932020-2-142-159.

Article received 24.01.2020

Reference a JournalArtic: Kuropyatnyk, O. Integrated management of exhaust gases of marine diesel engines as a way to ensure their environmental performance // Herald of the Odessa national maritime university. 2020. 2(62), 142-159. DOI 10.47049/2226-1893-2020-2-142-159. 